\title{
INFLUÊNCIA DOS SALTOS VERTICAIS NA PERCEPÇÃO DA CARGA INTERNA DE TREINAMENTO NO VOLEIBOL
}

\author{
INFLUENCE OF VERTICAL JUMP IN THEPERCEPTION OF THE INTERNAL VOLLEYBALL TRAINING LOAD \\ INFLUENCIA DE LOS SALTOS VERTICALES EN LA PERCEPCIÓN DE LA CARGA INTERNA DE \\ ENTRENAMIENTO ENEL VOLEIBOL
}

Thiago Andrade Goulart Horta' (Profissional de Educação Física) Maurício Gattás Bara Filho' (Profissional de Educação Física) Renato Miranda' (Profissional de Educação Física) Danilo Reis Coimbra² (Profissional de Educação Física) Francisco Zacaron Werneck ${ }^{3}$ (Profissional de Educação Física)

1. Universidade Federal de Juiz de Fora, Faculdade de Educação Física e Desporto, Núcleo de Pesquisa sobre Controle da Carga de Treinamento, Juiz de Fora, MG, Brasil.

2. Universidade do Estado de Santa Catarina, Departamento de Ciências da Saúde, Centro de Ciências da Saúde e Esporte, Joinville, SC, Brasil. 3. Universidade Federal de Ouro Preto, Centro Desportivo, Laboratório de Estudos e Pesquisas do Esporte e Exercício, Ouro Preto, MG, Brasil.

\section{Correspondência:}

Av. Barão do Rio Branco, no 1.191, apto. 1506, Juiz de fora, MG, Brasil. 36035-000.

thiagogoulart198@yahoo.com.br

\section{RESUMO}

Introdução: No voleibol, o salto vertical é considerado um elemento de extrema importância no treinamento, pois é necessário durante as ações de levantamento, saque, bloqueio e ataque, condicionando a obtenção de importantes vantagens nas ações ofensivas (ataque) e defensivas (bloqueio), elementos fundamentais para a conquista dos pontos no jogo. Objetivo: Analisar a influência do salto vertical na quantificação da carga de treinamento no voleibol por meio do método da percepção subjetiva do esforço (PSE) da sessão. Métodos: Participaram do estudo 15 atletas do sexo masculino de uma equipe de voleibol. Foi realizada a quantificação dos saltos e da PSE de 30 sessões de treinamento. Resultados: Na análise por posição, a PSE apresentou correlação positiva com o número de saltos e se mostrou mais forte nos opostos $(r=0,44 ; p=0,001 ; n=57)$, seguida pelos ponteiros $(r=0,34 ; p<0,001 ; n=109)$ e centrais $(r=0,18 ; p=0,03 ; n=141)$. Conclusão: Conclui-se que o salto vertical de ataque exerce maior influência no valor final de PSE apontado pelos atletas nas sessões de treinamento.

Descritores: voleibol; treinamento físico; atletas.

\section{ABSTRACT}

Introduction: In volleyball, vertical jump is considered an extremely important element in training, since it is necessary during setting, serve, block, and attack, determining the achievement of important advantages in offensive (attack) and defensive (block) actions, fundamental elements for the achievement of points in the game. Objective: To analyze the influence of the vertical jump in the quantification of training load in volleyball through the method of the subjective perceived exertion (RPE) of the session. Methods: Fifteen male athletes from a volleyball team participated in the study. The quantification of jumps and RPE of 30 training sessions was performed. Results: In the analysis by position, the RPE was positively correlated with the number of jumps and was stronger in the opposites $(r=0.44$; $p=0.001 ; n=57)$, followed by the setters $(r=0,34 ; p<0,001 ; n=109)$ and middles $(r=0.18 ; p=0.03 ; n=141)$. Conclusion: It is concluded that the attack vertical jump has greater influence on the final value of RPE pointed out by the athletes in training sessions.

Keywords: volleyball; physical training; athletes.

\section{RESUMEN}

Introducción: En el voleibol, el salto vertical es considerado un elemento de extrema importancia en el entrenamiento, pues es necesario durante levantamiento, saque, bloqueo y ataque, condicionando la obtención de importantes ventajas en las acciones ofensivas (ataque) y defensivas (bloqueo), elementos fundamentales para la conquista de los puntos en el juego. Objetivo: Analizar la influencia del salto vertical en la cuantificación de la carga de entrenamiento en el voleibol por el método de percepción subjetiva del esfuerzo (PSE) de las sesiones. Métodos: Participaron del estudio 15 atletas masculinos del sexo masculino de un equipo de voleibol. Se realizó la cuantificación de los saltos y de la PSE de 30 sesiones de entrenamiento. Resultados: En el análisis por posición, la PSE se correlacionó positivamente con el número de saltos y se mostró más fuerte en los opuestos $(r=0,44 ; p=0,001 ; n=57)$, seguida de los punteros $(r=0,34 ; p<0,001 ; n=109)$ y centrales ( $r=0,18 ; p=0,03, n=141)$. Conclusión: Se concluye que el salto vertical de ataque ejerce una mayor influencia en el valor final de PSE señalado por los atletas en las sesiones de entrenamiento.

Descriptores: voleibol; entrenamiento físico; atletas.

\section{INTRODUÇÃO}

Atualmente o método da percepção subjetiva do esforço da sessão (PSE) proposto por Carl Foster ${ }^{1}$ vem se destacando no monitoramento da carga interna de treinamento em equipes de esportes coletivos, por ser uma ferramenta eficaz e de simples utilização ${ }^{2,3}$. A PSE representa um parâmetro subjetivo de avaliação medida após a sessão de exercícios que reflete a resposta psicofísica gerada e memorizada no sistema nervoso central, decorrente dos impulsos neurais eferentes provenientes do córtex motor ${ }^{4-6}$. Essa resposta, segundo Impellizerl et al. ${ }^{7}$, é chamada de carga interna de treinamento, e refere-se as adaptações induzidas pelo treinamento decorrentes do nível de estresse imposto ao organismo. 
No voleibol, o salto vertical é considerado um elemento de extrema importância no treinamento, pois é necessário durante as ações de levantamento, saque, bloqueio e ataque, condicionando a obtenção de importantes vantagens nas ações ofensivas (ataque) e defensivas (bloqueio), elementos fundamentais para a conquista dos pontos no jogo. Considerando a natureza tática das atividades de salto e a frequência com que elas ocorrem em uma partida típica, sua manifestação no jogo de voleibol é de fundamental importância ${ }^{8-11}$. Além disso, a habilidade de salto vertical é considerada uma medida de rendimento de atletas de voleibol que atuam em alto nível ${ }^{12-14}$.

Apesar dos diversos estudos envolvendo a capacidade de saltos no voleibol, a análise do seu comportamento e relação com a carga interna gerada no processo de treinamento da modalidade ao longo de uma temporada competitiva é escassa. Em nosso entendimento existem algumas questões a serem respondidas em relação ao salto vertical no voleibol. A influência do número de saltos verticais realizados na carga interna de treinamento ainda é desconhecida. O tipo de salto vertical realizado pode ter influência na PSE? As diferentes demandas de saltos exigidos pelas diferentes posições dos atletas teriam impacto na carga interna de treinamento?

Acreditamos que a resposta dessas questões auxiliaria de forma direta no controle das ações de salto vertical realizados pelos atletas de voleibol de alto rendimento em suas rotinas de treinamentos, com o intuito de proporcionar treinamentos de alta qualidade e segurança na prevenção de lesões. Dessa forma, o objetivo do presente estudo foi analisar a influência do número de saltos verticais na carga interna de treinamento de atletas de voleibol de diferentes posições.

\section{MATERIAL E MÉTODOS}

\section{Amostra}

Participaram do estudo quinze atletas profissionais (idade: $28 \pm$ 4 anos, peso: 95,2 \pm 4,9 Kg, estatura:196,1 \pm 4,2 cm e percentual de gordura: 11,6 $\pm 3,7 \%$ ) do sexo masculino de uma equipe de voleibol de alto rendimento participante da Superliga Nacional, competição mais importante da modalidade no país. O elenco constava de dois Levantadores, cinco Centrais, quatro atacantes de Ponta, dois Líberos e dois atacantes Opostos. Estes atletas disputavam competições oficiais por, no mínimo, cinco anos.

A proposta de estudo foi apresentada aos atletas e explicado os possíveis riscos envolvidos. Os procedimentos do estudo seguiram a Declaração de Helsinque e foi aprovado pelo Comitê de Ética da Universidade Federal de Juiz de Fora (parecer n 036159/2013). Todos os atletas atestaram a participação voluntária e permitiram a utilização e divulgação das informações assinando o termo de consentimento livre e esclarecido.

\section{Procedimentos}

Foram analisadas 30 sessões de treinamento não sucessivas, durante o período da temporada caracterizado como competitivo (logo após o período preparatório). O fato do período preparatório não ter sido analisado se justifica pela ênfase na preparação física dos atletas neste período, com a ocorrência de poucos saltos nos treinamentos. Durante a temporada a equipe participou de duas competições oficiais: campeonato estadual e a superliga masculina de Vôlei.

O período de filmagem das 30 sessões de treinamento consistia especificamente dos treinamentos técnico-táticos (bloqueio, precisão de ataque, precisão de levantamento, saque-passe e jogo simulado), sendo descartado da análise o tempo de aquecimento e volta à calma.

Após o período de filmagens das sessões de treinamento, foi iniciada a Análise de Movimento (AM) dos vídeos para quantificação dos saltos dos atletas. As análises foram feitas por um único avaliador (TAGH), e os saltos foram quantificados em valores absolutos individualmente para cada posição (com exceção dos Líberos, que por características de suas funções em quadra, não executam saltos verticais em quantidade significativa). Dessa forma, os saltos foram classificados por posição de atuação dos atletas no voleibol (levantador, oposto, ponta e central), por tipo de treinamento realizado (bloqueio, saque, tático/simulado, precisão de ataque, precisão de levantamento), e por tipo de salto realizado (salto de saque, salto de bloqueio, salto de ataque, e salto de levantamento). Os saltos foram todos contabilizados para uma planilha em folha e posteriormente transferidos para uma planilha Excel (Microsoft Office $\left.{ }^{\circledR}\right)$. Essa planilha também continha a duração total líquida da sessão de treinamento, o valor da PSE do treinamento e o valor da carga de treinamento (CT), produto da multiplicação da duração total da sessão pelo valor da PSE ${ }^{5}$.

\section{Instrumentos}

Para a filmagem das sessões de treinamento foi utilizada a câmera filmadora modelo Sony Handycam ${ }^{\circledR}$ DCR-SX22.

A percepção subjetiva do esforço (PSE) da sessão foi coletada como propõem o método de Foster et al. ${ }^{5}$. Ao final do treinamento, aproximadamente 30 minutos após o término, o atleta respondia ao avaliador a seguinte pergunta: "Como foi sua sessão de treinamento?" A resposta era fornecida por meio da escala CR10 adaptada de Borg, em que os escores variam de 0 a 10.

\section{Análise estatística}

Utilizou-se estatística descritiva (frequência, média e desvio padrão) das cargas de treinamento e do número de saltos verticais. A normalidade da distribuição dos dados foi testada pelo teste de Kolmogorov Smirnov. Devido à distribuição normal, a correlação entre a percepção subjetiva do esforço (PSE) com o número de saltos foi analisada por posição por meio do teste de correlação de Pearson. Foram utilizados os seguintes critérios na interpretação da magnitude da correlação (r) entre as variáveis: $<0$. I trivial, 0.1 a 0.3 fraca, 0.3 a 0.5 moderada, 0.5 a 0.7 forte, 0.7 a 0.9 muito forte e 0.9 a 1.0 quase perfeita ${ }^{15}$. Diferenças na PSE, na CT e no número de saltos realizados entre as diferentes posições foram analisadas pela ANOVA de 1 fator, seguida pelo post hoc de Tukey. Para a análise dos dados, utilizou-se o programa estatístico SPSS (v. 19, SPSS Inc, Chicago, IL), considerando nível de significância de 5\% ( $<<0,05)$.

\section{RESULTADOS}

Um total de 367 sessões individuais de treinamento (duração média de 113,2 \pm 19,6 minutos) foi observado durante o estudo. Cada atleta realizou em média 10,7 $\pm 12,6$ saltos de saque e $36,3 \pm 22,5$ saltos de bloqueio por sessão. Os atacantes (pontas, opostos e centrais) realizaram em média 32,1 \pm 17,4 saltos de ataque e os levantadores 80,1 $\pm 44,5$ saltos de levantamento por sessão. O número médio de saltos realizados por atleta foi de 87,2 $\pm 37,9$ saltos/sessão. A PSE média foi de 5,5 $\pm 1,7$ e a CT média foi de $627 \pm 226$ (U.A).

Analisando as 30 sessões de treinamento, nove diferentes tipos de treinamento foram identificados (Tabela 1). O tipo de treinamento com o maior número de observações foi o "Tático + Precisão de Ataque", com 75 unidades de observações (U.O.). Na Tabela 1, são mostrados também os valores médios de PSE e o número de saltos nos diferentes tipos de treinamento técnico-táticos executados durante o período de treinamento analisado.

A Tabela 2 apresenta o número médio de saltos realizados por posição nas 30 sessões de treinamentos analisadas. Foi observada diferença significativa em relação ao número de saltos de saque $\left(F_{3,363}=10,369\right.$; $p<0,001)$, número de saltos de bloqueio $\left(F_{3,363}=27,283 ; p<0,001\right)$ e 
Tabela 1. Média \pm desvio-padrão da carga externa ( $n^{\circ}$ de saltos verticais) e PSE por tipo de treinamento (30 sessões).

\begin{tabular}{c|c|c|c}
\hline Tipo de treinamento & U.O. & $\mathbf{n}^{\circ}$ de saltos & PSE \\
\hline Bloqueio + Saque + Precisão de ataque & $\mathbf{n}$ & $\mathbf{( \overline { \mathbf { x } } \pm \mathbf { d p } )}$ & $\mathbf{( \overline { \mathbf { x } } \pm \mathbf { d p } )}$ \\
\hline Bloqueio + Precisão de levantamento & 13 & $33 \pm 24,35,9$ & $4,5 \pm 1,1$ \\
\hline Bloqueio & 13 & $36,9 \pm 13,7$ & $5,8 \pm 0,9$ \\
\hline Bloqueio + Tático & 25 & $113,4 \pm 23,4$ & $4,3 \pm 1,2$ \\
\hline Bloqueio + Tático + Precisão de ataque & 36 & $124,7 \pm 46,7$ & $5,7 \pm 2,0$ \\
\hline Tático & 50 & $78,3 \pm 32,9$ & $5,9 \pm 2,0$ \\
\hline Saque + Tático + Precisão de ataque & 71 & $87,2 \pm 27,9$ & $5,7 \pm 1,4$ \\
\hline Saque + Tático & 72 & $74,5 \pm 27,1$ & $5,2 \pm 1,5$ \\
\hline Tático + Precisão de ataque & 75 & $94,7 \pm 35,3$ & $6,0 \pm 1,6$ \\
\hline
\end{tabular}

U.O: Unidades de Observaçăo = número de atletas analisados x número de treinamentos por tipo de treino ocorrido nas 30 sessões analisadas; $\bar{x} \pm d p$ : média \pm desvio padrão; PSE: Percepção Subjetiva do Esforço.

Tabela 2. Média \pm desvio-padrão da carga externa ( $n^{\circ}$ de saltos verticais) de 30 sessões de treinamento por posição de jogo.

\begin{tabular}{c|c|c|c|c|c}
\hline Posições & $\begin{array}{c}\text { Saltos de } \\
\text { ataque } \\
(\overline{\mathbf{x}} \pm \mathbf{d p})\end{array}$ & $\begin{array}{c}\text { Saltos de } \\
\text { levantamento } \\
(\overline{\mathbf{x}} \pm \mathbf{d p})\end{array}$ & $\begin{array}{c}\text { Saltos de } \\
\text { bloqueio } \\
(\overline{\mathbf{x}} \pm \mathbf{d p})\end{array}$ & $\begin{array}{c}\text { Saltos de } \\
\text { saque } \\
(\overline{\mathbf{x}} \pm \mathbf{d p})\end{array}$ & $\begin{array}{c}\text { Total de } \\
\text { saltos } \\
(\overline{\mathbf{x}} \pm \mathbf{d p})\end{array}$ \\
\hline Levantador & - & $80,7 \pm 44,6$ & $25,1 \pm 17,1$ & $12,6 \pm 13,6$ & $119,2 \pm 48,5^{*}$ \\
\hline Oposto & $34,6 \pm 16,9$ & - & $29,0 \pm 17,5$ & $9,8 \pm 12,4$ & $73,5 \pm 24,0$ \\
\hline Ponteiro & $31,3 \pm 15,6$ & - & $30,8 \pm 17,5$ & $5,7 \pm 7,6^{*}$ & $67,8 \pm 24,5$ \\
\hline Central & $31,7 \pm 19,0$ & - & $48,3 \pm 24,5^{*}$ & $14,1 \pm 14,1$ & $94,1 \pm 34,9$ \\
\hline *Diferenças significativas $(\mathrm{p}<0,05)$ & entre as medidas; $\bar{x} \pm d p:$ média \pm desvio padrão.
\end{tabular}

número total de saltos $\left(\mathrm{F}_{3,363}=35,663 ; \mathrm{p}<0,001\right)$. O número de saltos de saque foi menor nos ponteiros comparados aos levantadores $(p=0,003)$ e centrais $(p<0,001)$. Os centrais realizaram maior número de saltos de bloqueio comparados aos levantadores, ponteiros e opostos $(p<0,001)$. Os levantadores realizaram o maior número total de saltos comparados às demais posições, seguidos pelos centrais, não havendo diferença significativa entre ponteiros e opostos. O número de saltos de ataque foi similar entre centrais, ponteiros e opostos $\left(F_{2,304}=0,716 ; p=0,49\right)$.

Foi observada diferença significativa na PSE entre as posições de jogo $\left(F_{3,363}=16,408 ; p<0,001\right)$. Os ponteiros e opostos apresentaram maior PSE quando comparados aos levantadores e aos centrais (Figura 1). O mesmo resultado foi observado em relação à $C T\left(F_{3,363}=11,261\right.$; $p<0,001$ ) (Figura 2).

A PSE dos levantadores não apresentou correlação com o número de saltos realizados durante as sessões de treinamento $(p>0,05)$. Já nos atacantes (opostos, centrais e pontas), a PSE se correlacionou positivamente com o número de saltos de ataque $(r=0,26 ; p<0,001$; $n=307$; fraca). Na análise por posição, essa correlação se mostrou mais forte nos opostos ( $r=0,44 ; p=0,001 ; n=57$; moderada), seguido pelos pontas ( $r=0,34 ; p<0,001 ; n=109$; moderada) e centrais $(r=0,18 ; p=0,03 ; n=141 ;$ fraca). A PSE dos opostos também se correlacionou positivamente com o número total de saltos $(r=0,30$; $p=0,02 ; n=57 ;$ moderada).

\section{DISCUSSÃO}

O presente estudo objetivou analisar a influência do número de saltos verticais na carga interna de treinamento de atletas de voleibol de diferentes posições. Stanganelli et al. ${ }^{16}$ utilizando a mesma abordagem metodológica do presente estudo reportaram valores semelhantes de saltos de ataque e bloqueio para as posições de Centrais e Ponteiros. No presente estudo, foi observado que o número de saltos verticais para execução do ataque no voleibol exerce influência na PSE dos atacantes, com maior impacto para os opostos e pontas apesar das correlações apresentadas serem consideradas de moderada magnitude. Bara Filho

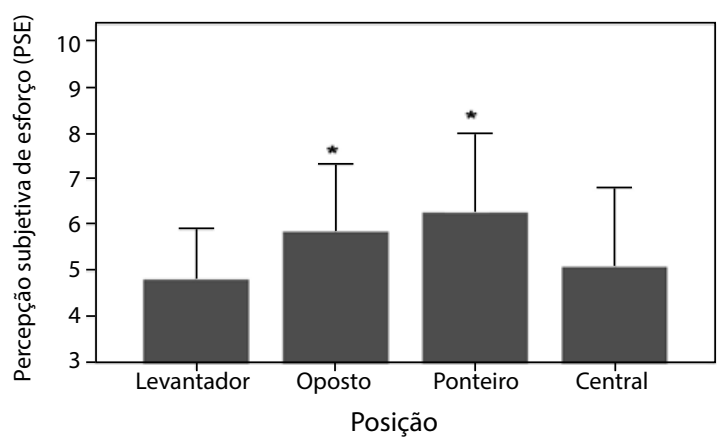

Figura 1. Comparação da Percepção Subjetiva do Esforço (PSE) das 30 sessões de treinamento analisadas por posições em quadra (média \pm desvio padrão).

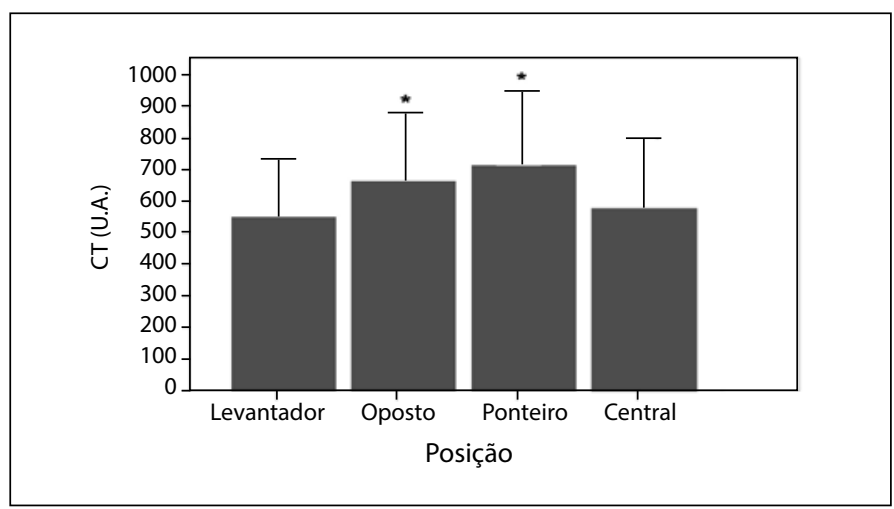

Figura 2. Comparação da Carga de Treinamento (CT) das 30 sessões de treinamento analisadas por posições em quadra de jogo (média \pm desvio-padrão).

et al. ${ }^{10}$, comparando diferentes métodos de controle da carga interna de treinamento em atletas profissionais de voleibol encontraram correlações positivas entre o método PSE de Foster e o método de Edwards por Zonas de Frequência CardíacaTRIMP $(r=0,45-0,67)$, resultado que reforça a praticidade e eficácia do método PSE para o controle de carga no voleibol de alto rendimento.

Por outro lado, Pereira et al. ${ }^{17}$ analisaram a relação do número de saltos verticais realizados em um teste de resistência de saltos com diferentes intervalos de recuperação e sua influência nos valores apontados de PSE com praticantes de voleibol e basquetebol. Diferente dos resultados encontrados em nosso estudo, altas correlações foram encontradas entre os números de saltos realizados e os valores de PSE $(r=0,97-0,99)$ em todas as baterias de testes com diferentes intervalos entre os saltos $(4 s, 5 s, 6 s)$. A PSE se correlacionou positivamente com a FC $(r=0,93-0,97)$ corroborando com outros estudos realizados em equipes de esportes coletivos que investigaram a relação do método da PSE com métodos baseados na FC'10,18-21.

Segundo Freitas et al. ${ }^{22}$, fatores como a capacidade física e a função que o atleta exerce na equipe podem influenciar diretamente na dinâmica da carga interna de treinamento. Foi mostrado que os atletas da posição de Ponta e Oposto apresentaram maiores valores de PSE com diferença estatística significativa em relação às outras posições durante as sessões de treinamento analisadas. Entretanto, as duas posições de atuação foram as que menos realizaram saltos verticais durante as sessões de treinamento. Os Ponteiros, por exemplo, são responsáveis juntamente com o Líbero pela recepção da primeira bola, tarefa que apresenta alto grau de execução técnica. Por outro lado, os Opostos realizam ataques com corridas de aproximação mais extensas, saltando frequentemente por trás da linha de três metros e recebendo alto volume de bolas de contra-ataque em uma partida $16,23,24$ 
Resultado interessante foi observado com o valor da PSE média encontrada ao longo de todas as sessões de treinamento analisadas $(5,5 \pm 1,7)$. De acordo com estudo de Lovell et al. ${ }^{20}$ que analisaram a validade do método PSE no monitoramento da carga de treinamento em atletas de rugby, o valor de PSE encontrado em nosso estudo está classificado como de intensidade moderada que engloba valores de 4 a 7 de PSE. Resultado semelhante foi reportado por Borin et al. ${ }^{25} \mathrm{com}$ um grupo de atletas de voleibol feminino. Corroborando com o resultado apresentado no presente estudo, porém utilizando a Frequência Cardíaca como parâmetro de avaliação, Stanganelli et al. ${ }^{16}$ e Bara Filho et al. ${ }^{10}$ também reportaram valores médios de FC classificados na zona moderada de intensidade ao decorrer de sessões de treinamento com atletas de voleibol de alto rendimento.

Em contrapartida, de acordo com Stagno et al. ${ }^{26}$ e Bara Filho et al. ${ }^{10}$, métodos de controle de carga de treinamento baseados em valores de FC podem não refletir a intensidade de atividades de curta duração e alta intensidade como em treinamentos que exijam esforços de características predominantes do sistema neuromuscular e com intervalos entre as execuções, como é característico no voleibol, não sendo a FC neste tipo de atividade o melhor parâmetro de controle.

Em relação aos tipos de treinamentos técnico-táticos realizados, os maiores valores médios de PSE foram encontrados nos treinamentos Tático e Tático + Precisão de Ataque. Tais achados corroboram com os encontrados por Bara Filho et al. ${ }^{27}$ quantificando e comparando a carga de diferentes tipos de treinamento em uma equipe profissional de Futebol. $\mathrm{O}$ treinamento Tático pode ser considerado como a reprodução de situações reais de jogo as quais o atleta enfrentará durante uma partida típica. De acordo com os autores, métodos de treinamento que se aproximem da realidade do jogo e que propiciem, concomitantemente, o desenvolvimento de diferentes capacidades físicas, técnicas, táticas e mentais devem ser priorizadas em busca da melhora da qualidade dos treinamentos, e consequentemente da melhora do desempenho do atleta.

Este estudo possui algumas limitações, como por exemplo, a não utilização de outros métodos de controle da carga de treinamento como os citados anteriormente em outros estudos. Ao considerar os constantes deslocamentos em alta intensidade que os atletas executam para a realização das ações nos treinamentos e partidas oficiais, acreditamos que o uso de ferramentas de quantificação de movimento, como o GPS (Sistema de posicionamento global), poderia registrar importantes informações em relação à demanda física imposta aos atletas de voleibol.

\section{CONCLUSÕES}

Conclui-se que o número de saltos verticais assim como as características de saltos exerce influência na carga interna de treinamento de atletas de voleibol de diferentes posições. Entretanto, o salto vertical isoladamente pode não refletir o estresse imposto ao organismo do atleta de maneira geral. Essa informação pode ser útil na programação e aplicação das cargas no processo de treinamento ao longo da temporada. Além disso, o método de percepção subjetiva do esforço da sessão conhecido como PSE mostrou-se um método prático, de simples utilização e eficaz em verificar o nível de carga interna imposta pelas cargas externas no processo de treinamento.

Todos os autores declararam não haver qualquer potencial conflito de interesses referente a este artigo.

CONTRIBUIÇÕES DOS AUTORES: Cada autor contribuiu individual e significativamente para o desenvolvimento deste manuscrito. TAGH (0000-0001-5183-9031)*, contribuiu substancialmente na redação do manuscrito e concepção do trabalho. MGBF (0000-0003-1219-8379)*, participou da aprovação final da versão e contribuiu substancialmente na concepção do trabalho e revisão crítica do conteúdo intelectual. DRC (0000-0002-6055-0667)* contribuiu substancialmente na concepção do trabalho e revisão crítica do conteúdo intelectual. FZW (0000-0003-1966-8820**, realizou a análise estatística dos dados e interpretação dos resultados. RM (0000-0002-1127-8513)*, participou da aprovação final da versão e revisão crítica do conteúdo intelectual no manuscrito. *ORCID (Open Researcher and Contributor ID).

\section{REFERÊNCIAS}

1. Foster C, Daines $E$, Hector L, Snyder AC, Welsh R. Athletic performance in relation to training load. Wis Med J. 1996;95(6):370-4.

2. Moreira A, Freitas CG, Nakamura FY, Aoki MS. Percepção de esforço da sessão e a tolerância ao estresse em jovens atletas de voleibol e basquetebol. Rev Bras Cineantropom Desempenho Hum. 2010;12(5):345-51.

3. Borin JP, Gomes AC, Leite GS. Preparação desportiva: aspectos do controle da carga de treinamento nos jogos coletivos. Rev Educ Fís/UEM. 2007;18(1):97-105.

4. Lambert MI, Borresen J. Measuring training load in sports. Int J Sports Physiol Perform. 2010;5(3):406-11.

5. Foster C, Florhaug JA, Franklin J, Gottschall L, Hrovatin LA, Parker S, et al. A new approach to monitoring exercise training. J Strength Cond Res. 2001;15(1):109-15.

6. Nakamura FY, Moreira A, Aoki MS. Monitoramento da carga de treinamento: a percepcao subjetiva do esforço da sessão é um método confiável? Rev Educ Fís/UEM. 2010;21(1):1-11.

7. Impellizzeri FM, Rampinini E, Marcora SM. Physiological assessment of aerobic training in soccer. J Sports Sci. 2005;23(6):583-92.

8. Sheppard JM, Cronin JB, Gabbett TJ, McGuigan MR, Etxebarria N, Newton RU. Relative importance of strength, power, and anthropometric measures to jump performance of elite volleyball players. J Strength Cond Res. 2008;22(3):758-65.

9. Sheppard JM, Gabbett TJ, Stanganelli LC. An analysis of playing positions in elite men's volleyball: considerations for competition demands and physiologic characteristics. J Strength Cond Res. 2009;23(6):1858-66.

10. Bara Filho MG, Andrade FC, Nogueira RA, Nakamura FY. Comparação de diferentes métodos de controle da carga interna em jogadores de voleibol. Rev Bras Med Esporte. 2013;19(2):143-6.

11. Felicissimo CT, Dantas JL, Moura ML, Moraes AC. Respostas neuromusculares dos membros inferiores durante protocolo intermitente de saltos verticais em voleibolistas. Motriz: Rev Educ Fis. 2012;18(1):153-64.

12. Rocha MA, Barbanti VJ. Análise das ações de saltos de ataque, bloqueio e levantamento no voleibol feminino. Rev Bras Cineantropom Desemp Hum. 2007;9(3):284-90.

13. Sheppard JM, Gabbett T, Taylor KL, Dorman J, Lebedew AJ, Borgeaud R. Development of a repeated-effort test for elite men's volleyball. Int J Sports Physiol Perform. 2007;2(3):292-304.

14. Sheppard JM, Mcguigan MR, Newton RU. The effects of depth-jumping on vertical jump performance of elite volleyball players: an examination of the transfer of increased stretch-load tolerance to spike jump performance. J Austr Strenght Cond. 2008;16:3-11.
15. Hopkins WG, Marshall SW, Batterham AM, Hanin J. Progressive statistics for studies in sports medicine and exercise science. Med Sci Sports Exerc. 2009;41(1):3-13.

16. Stanganelli LC, Dourado AC, Oncken P, Mançan S, da Costa SC. Adaptations on jump capacity in Brazilian volleyball players prior to the under-19 World Championship. J Strength Cond Res. 2008;22(3):741-9.

17. Pereira G, Correia R, Ugrinowitsch C, Nakamura F, Rodacki A, Fowler N, et al. The rating of per ceived exertion predicts intermittent vertical jump demand and performance. J Sports Sci. 2011;29(9):927-32

18. Alexiou H, Coutts AJ. A comparison of methods used for quantifying internal training load in women soccer players. Int J Sports Physiol Perform. 2008;3(3):320-30.

19. Impellizzeri FM, Rampinini E, Coutts AJ, Sassi A, Marcora SM. Use of RPE-based training load in soccer Med Sci Sports Exerc. 2004;36(6):1042-7.

20. Lovell TW, Sirotic AC, Impellizzeri FM, Coutts AJ. Factors affecting perception of effort (session rating of perceived exertion) during rugby league training. Int J Sports Physiol Perform. 2013;8(1):62-9.

21. Nunes JA, Costa EC, Viveiros L, Moreira A, Aoki MS. Monitoramento da carga interna no basquetebol. Rev Bras Cineantropom Desemp Hum. 2011;13(1):67-72.

22. Freitas VH, Miloski B, Bara Filho MG. Monitoramento da carga interna de um período de treinamento em jogadores de voleibol. Rev Bras Educ Fís Esporte. 2015;29(1):5-12

23. César B, Mesquita I. Caracterização do ataque do jogador oposto em função do complexo do jogo, do tempo e do efeito do ataque: estudo aplicado no voleibol feminino de elite. Rev Bras Educ Fís Esporte. 2006;20(1):59-69.

24. Sheppard JM. The competitive, physiological and trainable aspects of vertical jump performance: series of studies on the strength and conditioning of elite volleyball players [thesis]. Joondalup, Western Australia: Edith Cowan University, Faculty of Computing, Health and Science; 2008.

25. Borin JP, Dias RG, Leite GS, Padovani CRP, Padovani CR. Indicadores de desempenho e percepção subjetiva de esforço entre técnico e atletas de voleibol. Braz J Biomotr. 2010;4(2):123-30.

26. Stagno KM, Thatcher R, van Someren KA. A modified TRIMP to quantify the in-season training load of team sport players. J Sports Sci. 2007;25(6):629-34.

27. Bara Filho M, Matta M, Freitas DS, Miloski B. Quantificação da Carga de Diferentes Tipos de Treinamento no Futebol. Rev Educ Fís/UEM. 2011;22(2):239-46 